\title{
Engagement of general practitioners in falls prevention and referral to occupational therapists
}

\author{
Anne Mclntyre ${ }^{1}\left(\mathbb{D}\right.$, Lynette Mackenzie $^{2}$ (I) and Michele Harvey ${ }^{3}$ (D)
}

\begin{abstract}
Introduction: Falls prevention is a key activity for general practitioners, occupational therapists and other allied health professionals. Despite evidence for multidisciplinary community-based interventions, uptake of falls prevention is variable. General practitioners are crucial in identifying older people at risk of falls and referring for intervention.

This research aims to identify the current practice of general practitioners in falls prevention and to identify opportunities for occupational therapists in particular to extend their role in falls prevention in primary care.

Method: A total of 4000 paper surveys were mailed to randomly selected general practitioners within 209 clinical commissioning groups in England. Online surveys were also distributed via clinical commissioning groups to approximately 3200 general practitioners. Descriptive analysis of the data was undertaken.

Results: A total of 152 general practitioners responded. More than half indicated they were familiar with the UK guidelines for falls risk screening, but less than a third implemented these. Only $31 \%$ routinely asked older people if they had fallen. Whilst $90 \%$ identified occupational therapists as providing evidence-based falls prevention, only $74 \%$ referred to occupational therapy.

Conclusion: There are gaps in general practitioners' falls prevention referral practices to occupational therapists and allied health professionals. Better general practitioner pathways could enhance the quality of falls-prevention care for older people at risk.
\end{abstract}

Keywords

Accidental falls, falls prevention, general practitioners, survey, occupational therapy

Received: 11 January 2018; accepted: 13 September 2018

\section{Background}

Although people are living longer in the United Kingdom (UK), healthy life expectancy (71 years) is 10 years less than average life expectancy (81 years) (World Health Organization, 2017). Falls in older people may contribute to a threat to healthy life expectancy and are perceived as one of the 'giants' of health and social care (Close, 2005). Each year, $30 \%$ of people aged over 60 years and $50 \%$ of people aged over 80 years fall at least once (Public Health England, 2017), but this may be a conservative estimate as many falls are unreported (Martin, 2009). Consequences of falls affect the individual, their families and services. Fallers report physical injury that may be serious and the need to seek medical help (Stel et al., 2004). Logan et al. (2010) identified that $10 \%$ of all calls to UK ambulance services were for older people who had fallen; however, less than half of these were taken to hospital. Nevertheless, approximately 255,000 people aged $65+$ years are admitted to hospital due to falls in England each year, and nearly $7 \%$ of these never return home (Logan et al., 2010; Public Health England, 2017). Injurious falls are the fifth leading cause of death in older people (National Institute for Health and Clinical Excellence (NICE), 2013). Psychological consequences of falls include loss of autonomy, loss of self-efficacy and fear of falling (Lord et al., 2007). These consequences are experienced by the individual themselves and their families, whether associated with injury or not (Viera et al., 2016). A decline of everyday activity (by $35 \%$ ) and social participation $(15 \%)$ is also a reported consequence of falling (Stel et al., 2004). In monetary terms it is estimated that falls cost the public health system in the UK $£ 2.3$ billion each year (NICE, 2013). Therefore, the incidence and consequences of falls are a major public health concern.

Multiple risk factors are identified for falls, and the number and interaction of these factors increase the risk of falling (American Geriatric Society and British Geriatric Society, 2011). As the majority of older people aged $65+$ years have two or more long term health

\footnotetext{
${ }^{1}$ Senior Lecturer, Clinical Sciences, Brunel University, London, UK ${ }^{2}$ Associate Professor, Discipline of Occupational Therapy, University of Sydney, Sydney, Australia

${ }^{3}$ Research Assistant, Chelsea Children's Therapy, London, UK

Corresponding author:

Lynette Mackenzie, Discipline of Occupational Therapy, University of Sydney, 75 East Street, Lidcombe, NSW 2141, Australia.

Email: Iynette.mackenzie@sydney.edu.au
} 
conditions (Barnett et al., 2012), such multi-morbidity adds further complexity to falls prevention and intervention, requiring a holistic and multifactorial approach.

Research evidence demonstrates that falls are preventable, through identification of risks during everyday assessment and multifactorial intervention (Gillespie et al., 2015). The latest Cochrane systematic review of community-based fall interventions (Gillespie et al., 2012), systematic reviews and meta-analyses demonstrate continued support for balance and strength exercises, home safety interventions and medication reviews as effective in preventing falls in community-living older people (Clemson et al., 2008; Pit et al., 2007; Sherrington et al., 2008). As a result, NICE (2015) provides clear guidance and quality standards for assessment, and intervention based upon such research evidence, to prevent the first fall in community-living older people and to protect against subsequent falls. Indeed, the College of Occupational Therapists (2015) produced NICE accredited, evidence-based guidelines for falls management and prevention specifically for occupational therapists. Public Health England (PHE) (2017) also provides a consensus statement to support the commissioning of services for falls prevention by local commissioning and strategic leads in England.

Despite evidence for effective falls-prevention intervention and clear guidelines for practitioners, falls rates continue to increase (Gillespie et al., 2015). Uptake of falls prevention by older people themselves is variable, with older people often not perceiving themselves as 'fallers', even with a history of falls (Yardley et al., 2006). Dickinson et al. (2011) identified that older people do not report falls to health professionals, perceiving their falls to not be relevant, sufficiently severe or important. Health professional endorsement (especially general practitioner (GP) endorsement) increases uptake of services by patients (Roland et al., 2012). Therefore, GPs are crucial for identifying older people at risk of falls and referring them for intervention. However, studies in Australia and the United States (US) indicate that few older people are asked by their GP about falls or are offered interventions to prevent falls (Kielich et al., 2017). A study by Sturmberg (2002) highlighted that when at-risk populations attended their general practice, the risk of falls was identified in $50 \%$ of patients, with Wenger et al. (2003) ascertaining that routine screening increased the identification of fallers threefold. Both of these studies support early identification of falls risk in the primary care setting.

Primary health care approaches may be an effective falls-prevention strategy, but these require active engagement from GPs in collaboration with Allied Health Professionals (AHPs), particularly occupational therapists and physiotherapists (PTs) in the community. GPs are best placed to identify early falls risk in their older patients because they are responsible for the care of the majority of older people in the community and are referral agents for other community services such as occupational therapy and physiotherapy.

For community falls-prevention knowledge translation to take place, an understanding of the contributions of health professionals is needed (Damschroder et al., 2009). Kielich et al. (2017) identified that although Australian GPs recognised a previous fall as an important risk factor, they did not routinely ask their patients about falls, and their referral rates to AHPs were low. Middlebrook and Mackenzie (2012) reported that GPs had a poor understanding of what AHPs could offer in falls prevention, which influenced the low uptake of fallsprevention interventions by older people themselves. Other evidence suggests that when GPs do address falls, few based their practice on recognised clinical guidelines (Jones et al., 2011). Several barriers are faced by GPs in providing evidence-based care, including referrals to AHPs. These include lack of time during consultations, other more pressing issues, a lack of educational materials, organisational barriers, difficulty for GPs in initiating the process (Preen et al., 2006) and a lack of understanding of what allied health professionals can offer (Wilson et al., 2004).

Ganz et al. (2008) suggest that 'it takes a village to prevent falls' and argue that instead of the multiple providers of falls-prevention interventions working in isolation from one another, a more co-ordinated approach where all contributions are valued would be more effective in reducing falls and falls risk. This is especially important for services related to exercise, home evaluation and medications. As a key co-ordinator of services, the fallsprevention activity by GPs in the UK, including referral and screening practices, and GP knowledge of available services, is currently unknown. However, GP understanding about falls prevention will influence referrals made to occupational therapists, PTs and other AHPs, and their inclusion in a package of care. Further education and support for GPs in this role may be needed, especially knowing who to refer to in the challenging area of falls prevention for older people. The literature is clear about the need for a range of AHPs to be involved in falls prevention, such as occupational therapists (Leland et al., 2012; Peterson et al., 2012), PTs (Sherrington and Tiedemann, 2015), podiatrists (Wesley, 2011) and pharmacists (Laliberté et al., 2013). GP referrals to AHPs are one way to ensure that older people at risk of falls are linked to the services they need to prevent falls.

Understanding UK GP perspectives about their contributions to falls prevention, and exploring the potential issues related to the use of evidence in practice, are important to ensure older people have access to falls-prevention interventions in the community. This is especially pertinent with changes to the NHS England contract with GPs in 2014, which stated that all patients aged 75 years and over have a named accountable GP who ensures that they have a regular health check. The contractual agreement also stipulated that GPs should work with relevant health and social care professionals to deliver a multidisciplinary package of care that meets the needs of the older person (Health and Social Care Information Centre, 2014). This is even more significant with the recent PHE (2017) consensus statement on commissioning of local falls and fracture services. There seemed to be no clear model 
for engaging UK GPs in falls prevention, leaving a huge gap of missed opportunity. As there are contractual and operational differences in health care between the four countries of the UK, the focus of this study was to establish how GPs in England engage in falls-prevention assessment and referral to AHPs.

The study aims were to:

- Identify if and how English GPs address falls prevention in their routine practice with community-living older people;

- Identify GP understandings of falls risk factors and effective falls-prevention interventions;

- Investigate how GPs identify and/or screen older people at risk of falls;

- Examine GP referral practices to AHPs;

- Document any barriers or facilitators for GPs in implementing evidence about falls prevention in practice.

\section{Method}

\section{Pilot study}

Prior to the main study, a pilot online survey was adapted from the Kielich et al. (2017) Australian study. This was distributed by email to all the Clinical Commissioning Groups (CCGs) in NHS England $(n=213)$ and individual general practices listed on the NHS Choices website, supplemented by invitations distributed to CCGs through Twitter and LinkedIn sites. Only 37 responses were received using only online methods, and no changes were needed to the survey itself.

\section{Study advisors}

Older people living in the community and GPs were identified as key stakeholders and advisors for the study and made recommendations to the study team about the content and data collection methods for the study. Advisors were recruited using convenience sampling. A total of 13 older people advised the research team, including a faceto-face focus group of 10 older people. A predetermined topic guide was used for both individual interviews and the focus group. This confirmed the topics included in the survey and identified that very few of the older people had been asked about falls by their GP and most said that they would welcome more specific information about falls being provided by their GP. Five GPs participated in an individual interview or online focus group using GoToMeeting software $(n=4)$. These GPs described their local falls services as 'Cinderella' services. They felt that there was too much information provided to patients in their surgery and that they used their clinical reasoning to determine whether to ask their patients about incidence of falls. GPs indicated that paper questionnaires were more likely to enhance responses, as they felt overwhelmed by emails. However, they suggested that participants should be given an option for online completion. They recommended that the invitation should come via the practice manager or governance lead, but the invitation letter to the GP should be academically sound, informative and evidence-based. Simplicity of the survey with tick boxes to facilitate quick response, use of coloured paper to make the survey conspicuous and the use of incentives were also recommended. Knowing that this was a national survey with CCG endorsement was also perceived as important.

\section{Main study}

A cross-sectional questionnaire survey method was used 12 months after the pilot study. This allowed for concurrent information-gathering from GPs practising in England who were all subject to the same changes in GP contractual arrangements introduced by NHS England in 2014. Survey research enabled data to be collected from a representative sample of GPs in England (Kelley et al., 2003), the examination of GPs' perceptions and practices, and the identification of any educational requirements (Kramer et al., 2010).

The survey method of choice was to use paper surveys posted to participants, with endorsement by the GPs' clinical commissioning groups (CCGs). As other (monetary) incentives are known to improve response rates, an entry to a draw for a book token was offered.

Ethical approval was granted by Brunel University London and the University of Sydney. As a service evaluation, NHS Research Ethics Committee approval was not required. An information sheet about the study was provided to all participants and indicated that completion and return of the survey would imply that consent to participate was given.

\section{Participant recruitment}

GPs in current practice in England were identified as the study population, and support for the study was sought from CCGs. All 211 CCGs listed on the Health and Social Care website (now known as NHS Digital) were contacted by email via their CCG website. The email included information about the research, a link to the online version of the survey with a request for CCGs to distribute the online survey link to their GPs, as well as instructions on how to opt-in/out of the study. Four CCGs requested that their GPs should not be approached to participate in the study.

Proportionate sampling was utilised to select GPs for the paper survey from the remaining 207 CCGs. This ensured that the GPs likely to respond were more representative of the total population for each CCG. Once the numbers of GPs per CCG were calculated, actual participants were randomly selected using a research randomiser (www.randomizer.org). Invitations were addressed to the GP practice manager. Four CCG English regions (London, Midlands and East, North, and South) distributed the links to the online survey directly to GPs in their areas. This was either by email or by inclusion in their weekly and monthly E-bulletins to GPs. 


\section{Data collection}

A total of 4000 surveys were posted, containing letters to the practice manager and GP, participant information sheet, the survey and a pre-paid return envelope. The total number was influenced by budgetary restrictions for the study. A (potential) further 3200 GPs were sent a link to the survey by 10 CCGs for online completion.

In order to enhance response rate and also to build research capacity within the team, $\mathrm{MH}$ undertook an online course on questionnaire design. This informed the structure and content of the survey.

The survey was developed based on literature related to international GP practice in falls prevention and current falls-prevention clinical guidelines. Survey topics included the perceptions, knowledge and routine practice of GPs in relation to identifying, screening and assessing falls risks, falls management and referral practices, and barriers and facilitators to effectively preventing falls in older patients. Answers required tick-box responses where possible, with few open-ended options. The survey contained 26 questions divided into four sections, with the first section collecting demographic data (10 questions), the second addressing GP falls-prevention practice for community-living older people (eight questions), the third addressing GP falls management and interventions (three questions), and the final section addressing community falls programmes and AHPs (five questions). The online version had the same content and format as the paper version but was constructed using the Bristol Online Survey (BOS) platform.

Participants (postal and online) could download an acknowledgement of participation for Continuing Professional Development purposes on completion of the survey. At this point, participants were also invited to enter the prize draw, where 20 prizes of $£ 50$ gift vouchers were offered to maximise the response rate.

\section{Data analysis}

Responses from paper surveys were manually entered into a copy of the BOS survey, downloaded and exported into SPSS20. Online responses were exported from BOS and downloaded as Excel spreadsheets and SPSS datasets. Quantitative data were summarised using descriptive statistics and regression analysis, and open-ended responses were analysed using descriptive, thematic analysis. Both $\mathrm{AM}$ and $\mathrm{MH}$ scrutinised and analysed the data and checked for any errors.

\section{Results}

A total of 152 surveys were completed and returned within the time frame. Of these, 85 were paper surveys and 67 online completions. Ten surveys were returned uncompleted saying GPs were 'too busy to complete', or that 'no-one willing to complete with no benefit'. This means that the response rate for the paper survey was a very low at $2 \%$. As the online survey potentially went to 3200 GPs, the response rate for this online survey can also be estimated at 2\%. Responses came from 33\% $(n=69)$ CCGs across England and all four CCG regions were represented. One CCG who gave the link in their E-Bulletin to GPs returned 36 responses $(23.4 \%$ of the total), with all but one survey from this CCG completed online. The respondents did not always complete every question of the survey, and percentages were calculated on the total number of answers given for each item.

\section{Demographics}

Most respondents $(36.1 \%)$ had been in practice for more than 20 years, with most practising in an urban area $(71.9 \%)$. Most responders were GP partners $(93.3 \%)$. The mean number of community-based patients aged over 65 on the responders' caseloads was $25.7 \%$ (ranging from $2-70 \%$ ). GPs saw a median of 50 older people per week (range 5-500) and estimated that a median of 10 (range 1-250) of these were at risk of falling.

\section{Addressing falls prevention in routine practice}

More than half of respondents said that they were familiar with the NICE (2015) guidance but less than a third stated that they implemented it in practice. When asked about screening for falls, a quarter said that this should occur annually, and a further $14.8 \% \quad(n=22)$ said that this should occur on every consultation or during a general health check $(47.7 \%, n=71)$. Others suggested falls screening should take place when a patient self-reported a fall or fear of falling $(55 \%, n=82)$ or in conjunction with other health-related consultations $(54.4 \%, n=81)$. A third of responders $(n=46)$ said that they routinely asked older patients if they had had a fall in the past year, with a similar number stating that they asked the circumstances of the older person's fall. Fewer $(14.1 \%)$ GPs $(n=21)$ asked about fear of falling. A further $10.1 \%(n=15)$ identified an older person at risk of falls if they had signs of frailty, complaints of dizziness, mobility problems or if the GP felt it was relevant. This included the GP being suspicious that a fall had occurred.

Regression analysis of GP characteristics (size of practice, gender, time in general practice, percentage of older people on their caseload) and the likelihood of them asking patients about falls during consultations did not reveal any significant results.

\section{Identifying and screening of falls risk}

The falls risk factor considered most important for GPs to address in practice was multiple medications $(97 \%$, $n=143)$, followed by gait and balance impairments $(91.9 \%, n=135)$, postural hypotension $(88.6 \%, n=130)$ and past falls history $(85.3 \%, n=128)$.

\section{GP identification and screening of falls risk}

Whilst $51.3 \%(n=77)$ of GPs indicated that they had carried out medical history reviews with older patients in the 


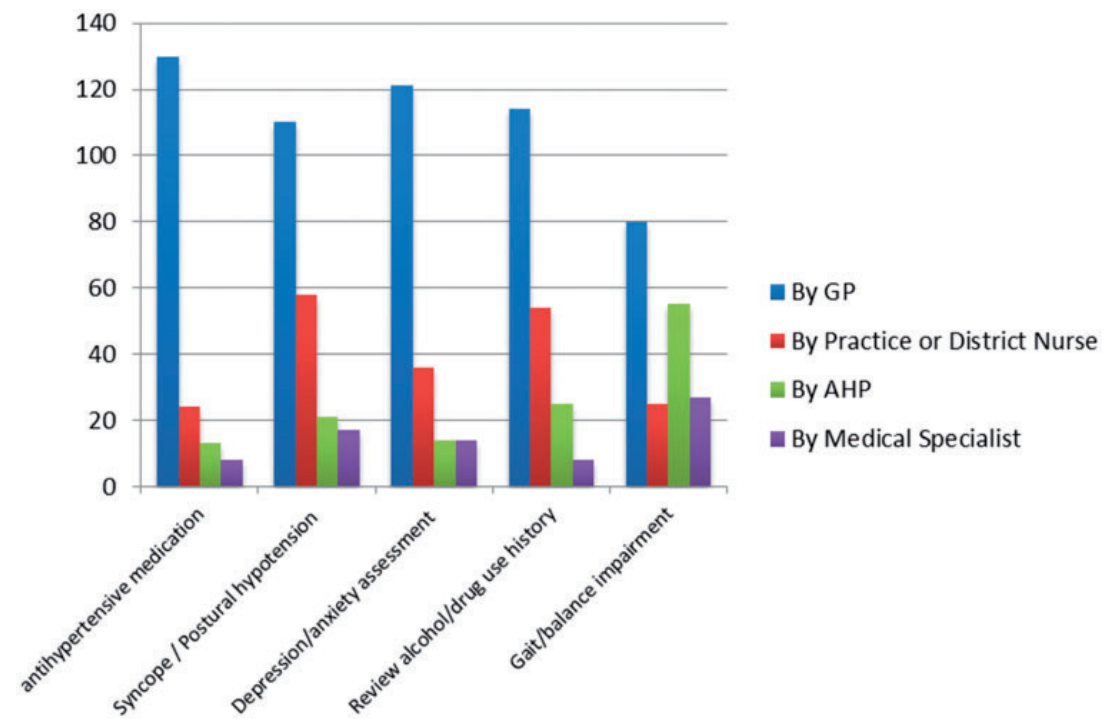

Figure 1. Assessments most likely to be carried out by GPs. AHP: allied health professional.

last year, more GPs $(86 \%, n=129)$ indicated that they would primarily carry these out. The most common assessments primarily carried out by GPs were all medicationrelated, for example assessment of multiple medications $(84.7 \%, \quad n=127)$, antipsychotic medication $(88.7 \%$, $n=133)$, use of sedatives $(90 \%, n=133)$, and antihypertensive medication $(86.7 \%, n=130)$.

Assessments least likely to be carried out by the GP practices were those that are associated with the role of other disciplines, such as mobility and gait assessments, home hazard and modification, and activities of daily living (see Figure 1). Some GPs attempted to carry out these assessments themselves or had these completed by practice nurses (see Figure 2).

When asked about what their first step would be in identifying a patient at risk, $56 \%$ of respondents $(n=84)$ said that they would want to conduct a more in-depth assessment themselves, with another $26.7 \%(n=40)$ identifying a referral to a falls service and only $8 \%(n=12)$ suggesting that they would make a direct referral to an AHP.

\section{GP referral practices to AHPs}

The three most necessary AHPs identified by GPs for evidence-based falls intervention were PT $(92.7 \%, n=139)$, occupational therapy $(92 \%, n=138)$ and podiatry $(64 \%$, $n=96)$. However, the number of referrals made annually to any AHPs varied between 0 and 600 (for a whole practice) with the median being 10. Most frequent referrals were to PT $(78.7 \%, n=118)$, followed by $75.3 \%$ $(n=113)$ to occupational therapy and $17.3 \%(n=26)$ to podiatry. Community falls services and clinics were referred to by $43 \%$ of GPs $(n=63)$.

When referring older people to AHPs for falls prevention, the survey results indicated that GPs relied on their own records and databases $(56 \%, n=86)$, their knowledge of local services or contacts $(25.3 \%, n=38)$ and the recommendation of colleagues $(16.6 \%, n=25)$. Few would look for information regarding falls-prevention services $(13.3 \%$ $n=20$ ) in local service directories or Internet searches.

\section{Barriers and facilitators to implementing falls prevention}

Although 14 GPs $(9.3 \%)$ said that they did not experience any barriers to implementing falls prevention, the most common barrier identified by GPs was lack of consultation time to ascertain risk and refer $(58 \%, n=87)$, followed by a perceived lack of local NHS AHPs to refer to $(34.7 \%$, $n=52$ ) (see Figure 3). Other barriers were that referrals to local falls services were too lengthy or complicated $(7.3 \%$, $n=11$ ), or that it was too difficult to integrate referrals into their practice and infrastructure $(17.3 \%, n=26)$. A small number $(6.7 \%, n=10)$ indicated that, where falls services existed, services struggled to cope with demand. Fifteen GPs $(10 \%)$ stated that not having falls assessment as part of the Quality Outcomes Framework (QOF) was a barrier to addressing falls. However, five $(3.3 \%)$ respondents suggested that GPs do not know how to identify appropriate patients in need of referral.

Qualitative data revealed both positive and negative comments about local falls services. Barriers to implementing falls prevention were compounded when falls services were perceived as not providing adequate solutions. Respondents also felt that GPs lacked the resources and training for falls prevention. Integrated and local falls services were seen to be a positive resource, especially with specialist practitioners.

\section{Discussion}

\section{Implications of results for primary health care provision for older people at risk of falls}

This study explored GP falls-prevention practice in England and the contextual factors that positively and 


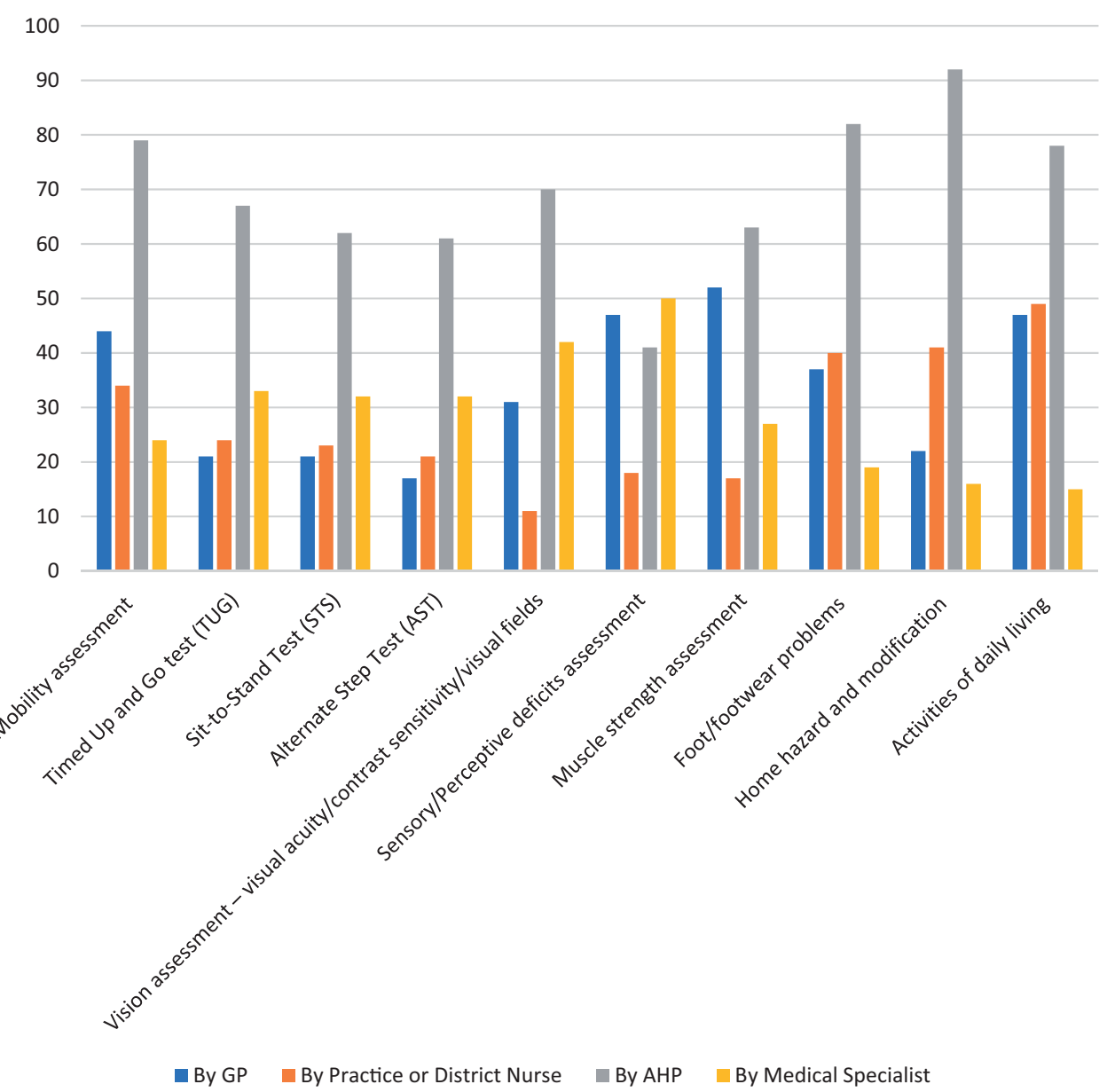

Figure 2. Assessments least likely to be carried out by GPs. AHP: allied health professional.

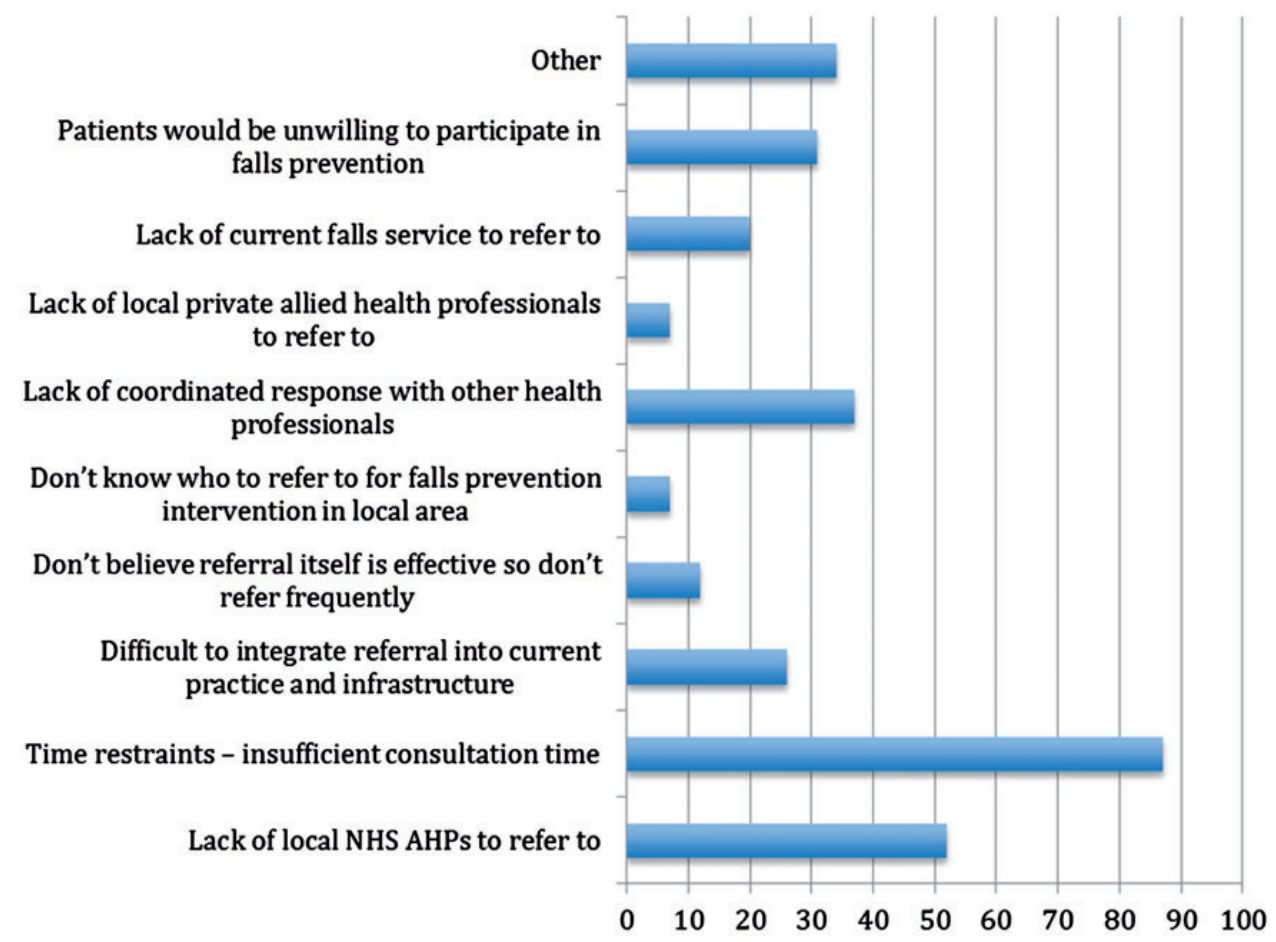

Figure 3. Barriers and facilitators to implementing falls prevention. 
negatively affect this activity. With such a low response rate to the survey $(2 \%)$, these findings cannot be generalised to the larger GP population in England, despite all CCG regions being represented. However, these response rates concur with evidence (Parkinson et al., 2015; Pit et al., 2014), the pilot for this study in NHS England and a survey of Australian GPs by Kielich et al. (2017).

There was inconsistency between GP familiarity with NICE (2013) guidance and its implementation. Although most GPs were familiar with the NICE (2013) guidelines, only a third said that they routinely asked about falls in a 12-month period, less than half said they implemented the guidelines and $20 \%$ identified lack of GP training in falls prevention as being a barrier in practice. Although most GPs said that they would screen for falls during a consultation, lack of time during consultation and addressing the more immediate demands of the patient were barriers to regularly screening for falls. These findings are similar to those of Preen et al. (2006) and Jones et al. (2011). Sturmberg (2002) and Wenger et al. (2003) found that asking older people about falls tended to be done by GPs with a higher number of older people at risk of falls attending their surgery. In our study this was not a significant finding. Inclusion of falls assessment in the QOF was suggested by GP advisors and some survey participants as a possible facilitator to increase falls risk assessment; however, this would not necessarily encourage the use of evidence-based pathways and decision-making by GPs.

GPs in our study identified multiple medications, gait and balance impairments, postural hypotension and past falls history as key issues for them to consider in relation to falls risk. According to a recent systematic review of predictors of falls (Lusardi et al., 2017), two of these are consistent with the conclusions of the review which identified five falls risks that should be considered (history of falls, use of psychoactive medications, requiring assistance for any activities of daily living (ADL), fear of falling and use of an ambulatory assistive device). Few of the GPs in our study asked about fear of falling, despite the prominence of this risk factor in the review.

Once an older person is identified as being at risk of falling by a GP, it appears that in-depth assessments would be carried out 'in-house'. Given the available expertise of AHPs in some of the assessments listed in the survey, it is surprising that many GPs attempted to carry out these assessments themselves. It may be that GPs referred to AHPs and medical specialists where they recognised that they lacked expertise. However, GPs in this study acknowledged the expertise of occupational therapists, PTs and podiatrists. GPs were more likely to refer to AHPs for assessment of activities of daily living, home hazards, mobility or for foot/footwear treatment, but some suggested they carried out these assessments themselves. These GPs may have preferred to rely on what (or who) they knew and could control, within their available resources (including time), because they found the referral processes, lack of local AHPs and falls services challenging. Whilst some GPs recognised the skills of
AHPs, unlike Wilson et al. (2004), GPs limited the access of older patients to AHPs. As GPs tended to conduct some assessments usually undertaken by AHPs, this may have reduced time available for their consultations.

It is worth noting that a few GPs were dissatisfied with the service offered by their local falls service provider, especially when the falls service seemed to only assess and refer back to the GP with recommendations for action rather than implementing interventions to address the referring problem/need. What is unknown here is whether there was a lack of understanding of the role of falls services or a lack of communication between service and GP. Some GPs stated that they did not know how to identify appropriate people to refer to falls services, and other GPs requested resources and training for GPs on falls prevention so that they could provide better falls assessment and management for their older patients. Conversely, other GPs stated that integrated and local falls services worked well.

\section{Methodological considerations}

As already stated, the very low response rate for this survey means that the findings cannot be generalised to the wider population of GPs practising in England. However, all CCG regions in England were represented within the data, and the data reflects the practice of 150 GPs. GP surveys frequently attract low response rates; for instance, one survey using an established database of GP contacts had a response rate of under $1 \%$, and following reminders only added another $2.4 \%$ (Parkinson et al., 2015). Therefore, as this study used a similar method, the results are consistent. Monetary incentives (which we used), using postal surveys, using a mixed method approach such as online followed by postal surveys, peer recruitment by telephone and using registered mail are strategies to improve response rates (Pit et al., 2014). Some of these methods threaten the anonymity of responses and are also costly to implement, and the best methods are still being debated. Both pilot study findings and feedback from GP advisors strongly advocated paper surveys posted to GPs to enhance response rate, as online surveys may not have been as effective. This feedback was consistent with research evidence from other researchers (Cottrell et al., 2015 ; Pit et al., 2014). However, online survey methods were also used as an option for GPs to complete and specifically requested by some CCGs. It is important to note that in this study, the response rates for both paper and online surveys were the same at $2 \%$. What is also of interest is that where one CCG advertised the survey and gave the link in their E-bulletin to GPs, the number of responses was the highest response from a CCG and nearly a quarter of all responses (online and paper) to the survey. This may have been due to the endorsement by the CCG to promote the survey or because GPs who read the E-bulletin were also more likely to respond in online format.

When considering the time and financial implications of online and paper surveys, online surveys are much more 
cost effective to administer (with greater opportunity to send out several reminders and invitations). Data management for analysis is also efficient as online survey software such as BOS allows for data downloading in a format ready for data analysis, unlike the paper surveys, which required manual data entry.

\section{Conclusion}

This study surveyed GPs in England about their engagement in falls prevention as part of their contributions to public health for older people. Recommendations can only apply to the participating GPs because of the low response rate of $2 \%$. However, these results reflect those of other studies in other countries with similar falls guidance and practice. The results from this study may inform GPs and AHPs and provide them with an opportunity to reflect upon their services. Time constraints seemed to be the major factor in preventing GPs from implementing falls risk assessment, prevention and referral as well as attempting to meet QOF targets. Including falls risk assessment as part of the QOF might increase the number of assessments of falls risk by GPs but would not necessarily encourage the use of evidence-based pathways and decision-making.

As GPs seem to rely on their own or colleagues' resources, promoting more localised and on-going personal connections between GPs and occupational therapists or other falls services might enhance referrals and GP understanding of falls prevention. Occupational therapists should use opportunities to report back to GPs about any of their patients who have received falls-prevention interventions (whether or not the GP initiated the referral). This would be a key way that occupational therapists could facilitate education for GPs about falls prevention and the services that occupational therapists can provide. The opportunity for training GPs about local falls services provision would enhance the timely and appropriate referral of older people at risk of falling to these services. Such communication would also facilitate the designing and commissioning of future local falls services as advocated by PHE (2017).

This paper adds to the methodological debate about survey research with GPs. The literature, our previous pilot study and GP advisors recommended the use of paper surveys to enhance response rate; however, there was no difference in the response rate for paper and online survey completion. Future research would benefit from active involvement and endorsement by CCGs on a national level, and localised research carried out by local occupational therapists and falls services with their GP colleagues would be informative. Further research is needed to inform health services planning and development.

\section{Key findings}

- Familiarity with falls guidelines was not matched by GP implementation and referral for falls prevention.

- Local research involving supportive CCGs might be more effective than national surveys of GPs.

\section{What the study has added}

This study has provided a clear outline of the perspective of GPs in falls prevention, and indicates that falls-prevention providers, including occupational therapists, should proactively work closely with GPs to enhance referrals for falls management for older people.

\section{Research ethics}

Ethical approval was obtained from Brunel University, London (Ref: 14/7/STF/18) 2015, and the University of Sydney (Ref: 2013/610).

\section{Consent}

All participants gave informed consent: they consented to participate in the online survey by clicking a box after the embedded participant information statement.

\section{Declaration of conflicting interests}

The authors declared no potential conflicts of interest with respect to the research, authorship and/or publication of this article.

\section{Funding}

The authors disclosed receipt of the following financial support for the research, authorship and/or publication of this article: Research Career Development Grant from the UK Occupational Therapy Research Foundation awarded in 2015

\section{Contributorship}

Anne McIntyre and Lynette Mackenzie researched literature, applied for ethical approval, and contributed to the development of the data collection methods and analysis plans. Anne McIntyre and Michele Harvey undertook the data collection. All authors contributed to the analysis, interpreted the data and contributed to the development of the manuscript. All authors reviewed and edited the manuscript and approved the final version.

\section{ORCID iD}

Anne McIntyre (D) http://orcid.org/0000-0003-1667-036X

Lynette Mackenzie (D) http://orcid.org/0000-0002-1597-2051

Michele Harvey (D) http://orcid.org/0000-0003-0958-7525

\section{References}

American Geriatrics Society and British Geriatrics Society. (2011) Summary of the updated American geriatrics society/ British geriatrics society clinical practice guideline for prevention of falls in older persons. Journal of the American Geriatrics Society 59(1): 148-157.

Barnett K, Mercer SW, Norbury M, et al. (2012) Epidemiology of multimorbidity and implications for health care, research, and medical education: A cross-sectional study. The Lancet 380(9836): 37-44.

Clemson L, Mackenzie L, Ballinger C, et al. (2008) Environmental interventions to prevent falls in communitydwelling older people: A meta-analysis of randomized trials. Journal of Aging and Health 20(8): 954-971.

Close JCT (2005) Prevention of falls in older people. Disability and Rehabilitation 27(18-19): 1061-1071.

College of Occupational Therapists (2016) Occupational Therapy in the Prevention and Management of Falls in Adults; Practice Guideline. London: RCOT. 
Cottrell E, Roddy E, Rathod T, et al. (2015) Maximising response from GPs to questionnaire surveys: Do length or incentives make a difference? BMC Medical Research Methodology 15: 3. DOI: 10.1186/1471-2288-15-3.

Damschroder L, Aron D, Keith R, et al. (2009) Fostering implementation of health services research findings into practice: A consolidated framework for advancing implementation science. Implementation Science 4: 50. DOI: 10.1186/1748-59084-50.

Dickinson A, Horton K, Machin I, et al. (2011) The role of health professionals in promoting the uptake of fall prevention interventions: A qualitative study of older people's views. Age and Ageing 40(6): 724-730.

Ganz D, Alkema G and Wu S (2008) It takes a village to prevent falls: Reconceptualizing fall prevention and management for older adults. Injury Prevention 14(4): 266-271.

Gillespie LD, Robertson MC, Gillespie WJ, et al. (2012) Interventions for preventing falls in older people living in the community. Cochrane Database of Systematic Reviews 12(9): CD007146.

Health and Social Care Information Centre (2014) General Practice Extraction Service (GPES): Benefits Plan. Named GP for Patients Aged 75 and over Quality Service 2014-15. London: HSCIC.

Jones TS, Ghosh TS, Horn K, et al. (2011) Primary care physicians' perceptions and practices regarding fall prevention in adult's 65 years and over. Accident Analysis and Prevention 43(5): 1605-1609.

Kelley K, Clark B, Brown V, et al. (2003) Good practice in the conduct and reporting of survey research. International Journal for Quality in Health Care 15(3): 261-266.

Kielich K, Mackenzie L and Lovarini M (2017) Urban Australian general practitioners' perceptions of falls risk screening, falls risk assessment, and referral practices for falls prevention: An exploratory cross-sectional survey study. Australian Health Review 41(1): 111-119.

Kramer BJ, Ganz DA, Vivrette RL, et al. (2010) Development of a fall prevention survey to determine educational needs for primary care providers. Gerontology \& Geriatrics Education 31(4): 310-327.

Laliberté M, Perreault S, Damestoy N, et al. (2013) The role of community pharmacists in the prevention and management of osteoporosis and the risk of falls: Results of a cross-sectional study and qualitative interviews. Osteoporosis International 24(6): 1803-1815.

Leland N, Elliott S, O'Malley L, et al. (2012) Occupational therapy in fall prevention: Current evidence and future directions. American Journal of Occupational Therapy 66(2): 149-160.

Logan PA, Coupland CAC, Gladman JRF, et al. (2010) Community falls prevention for people who call an emergency ambulance after a fall: Randomised controlled trial. BMJ 340: c2102. DOI: $10.1136 /$ bmj.c2102.

Lord S, Sherrington C, Menz H, et al. (2007) Falls in Older People, 2nd ed. Cambridge: Cambridge University Press.

Lusardi M, Fritz S, Middleton A, et al. (2017) Determining risk of falls in community dwelling older adults: A systematic review and meta-analysis using post-test probability. Journal of Geriatric Physical Therapy 40(1): 1-36.

Martin FC (2009) Next steps for falls and fracture reduction. Age and Ageing 38(6): 640-643.

Middlebrook S and Mackenzie L (2012) The enhanced primary care program and falls prevention: Perceptions of private occupational therapists and physiotherapists. Australasian Journal on Ageing 31(2): 72-77.

National Institute for Health and Clinical Excellence (2013) Falls: Assessment and Prevention of Falls in Older People (CG161). London: NICE. Updated 2014.

National Institute for Health and Clinical Excellence (2015) Falls in Older People. Quality Standard (QS86). London: NICE. Updated January 2017.

Parkinson A, Jorm L, Douglas K, et al. (2015) Recruiting general practitioners for surveys: Reflections on the difficulties and some lessons learned. Australian Journal of Primary Health 21(2): 254-258.

Peterson E, Finlayson M, Elliott S, et al. (2012) Unprecedented opportunities in fall prevention for occupational therapy practitioners. American Journal of Occupational Therapy 66(2): 127-130.

Pit S, Byles J, Henry D, et al. (2007) A quality use of medicines program for general practitioners and older people: A cluster randomised controlled trial. The Medical Journal of Australia 187(1): 23-30.

Pit S, Vo K and Pyakurel S (2014) The effectiveness of recruitment strategies on general practitioner's survey response rates - a systematic review. BMC Medical Research Methodology 14: 76.

Preen D, Bailey B and Wright A (2006) Willingness of general practitioners to participate in enhanced primary care discharge care planning. Medical Journal of Australia 184(2): $90-91$.

Public Health England (PHE) (2017) Falls and Fracture Consensus Statement: Supporting Commissioning for Prevention. London: Public Health England.

Roland M, Guthrie B and Thomé DC (2012) Primary medical care in the United Kingdom. Journal of the American Board of Family Medicine 25(Supp1): S6-S11.

Sherrington C and Tiedemann A (2015) Physiotherapy in the prevention of falls in older people. Journal of Physiotherapy 61(2): 54-60.

Sherrington C, Whitney JC, Lord SR, et al. (2008) Effective exercise for the prevention of falls: A systematic review and metaanalysis. Journal of the American Geriatrics Society 56(12): 2234-2243.

Stel VS, Smit JH, Pluijm SMF, et al. (2004) Consequences of falling in older men and women and risk factors for health service use and functional decline. Age and Ageing 33(1): 58-65.

Sturmberg J (2002) Health assessments in general practice. Australian Family Physician 31(4): 384-387.

Viera ER, Palmer RC and Chaves PHM (2016) Prevention of falls in older people living in the community. BMJ 353: i1419. DOI: $10.1136 /$ bmj.i1419.

Wenger NS, Solomon DH, Roth CP, et al. (2003) The quality of medical care provided to vulnerable community-dwelling older patients. Annals of Internal Medicine 139(9): 740-747.

Wesley V (2011) Prevention of falls through podiatry care. BMJ 342: d3052. DOI: 10.1136/bmj.d3052.

Wilson S, Marks R, Donohoe S, et al. (2004) General practitioner multidisciplinary skills for enhanced primary care. Australian Family Physician 33(6): 479-480.

World Health Organization (2017) World Health Statistics 2017: Monitoring Health for the SDGs, Sustainable Development Goals: Annex B. Geneva: WHO.

Yardley L, Bishop FL, Beyer N, et al. (2006) Older people's views of falls preventions in six European countries. The Gerontologist 46(5): 650-660. 